\title{
Relación entre la práctica de actividad física y el apoyo social percibido de los adolescentes de la provincia de Soria
}

\section{Relationship between the physical activity practice and the social support perceived in adolescents of the province of Soria}

\author{
Daniel Sanz-Martín ${ }^{1}$, Germán Ruiz-Tendero ${ }^{2 *}$ y Emilia Fernández-García ${ }^{2}$ \\ 1 CEIP Infantes de Lara, Soria (España). \\ 2 Universidad Complutense de Madrid (España).
}

\begin{abstract}
Resumen: Existe cierta preocupación social por conocer los niveles de práctica de actividad física de los adolescentes, para poder llevar a cabo propuestas de mejora si fuera necesario. Este estudio fue diseńado con la finalidad de conocer esos niveles de los adolescentes de Soria y el apoyo social que perciben para su práctica, tanto de familiares como de amigos, en función del sexo del alumnado, e identificar el tipo de relación existente entre su práctica y la percepción del apoyo social. La muestra estuvo formada por 694 adolescentes de Educación Secundaria Obligatoria de la provincia de Soria, a los cuales se les administró Four by one day physical activity questionnaire, parent support scale y peer support scale. Los resultados mostraron diferencias significativas en los niveles de actividad física y de apoyo social en función del sexo del alumnado y se obtuvo correlación significativa entre estos niveles y el apoyo percibido.

Palabras clave: Niveles de actividad física, apoyo de familia, apoyo de amigos y estudiantes.
\end{abstract}

Abstract: There is a social concern about knowing the levels of physical activity practice of adolescents, in order to carry out improvement proposals if necessary. This study was designed with the purpose of getting to know those levels of adolescents of Soria and the social support they perceive for their practice, from family and friends, depending on the gender of the students, and identifying the type of relationship between their practice and social support perceived. The sample selected was 694 adolescents of Compulsory Secondary Education from the province of Soria, to whom Four by one day physical activity questionnaire, parent support scale and peer support scale, were administered. The results showed significant differences in physical activity levels and social support according to the gender of the students and a significant correlation has been obtained between these levels and the support perceived.

Key words: Physical activity levels, family support, friends support and students.

\section{Introducción}

La preocupación social por la salud y el interés de su mejora a través de la práctica de actividad física (AF) son dos ámbitos prioritarios de actuación a nivel mundial, justificados en parte por la consideración de la inactividad física como el cuarto factor de riesgo más importante de mortalidad en el mundo (OMS, 2009) y la evidencia sobre los beneficios que aporta la práctica de actividad física (OMS, 2010).

Durante la adolescencia los jóvenes soportan numerosos cambios, no solo corporales, estando algunos relacionados con los estilos de vida, exponiéndose a conductas que implican un riesgo para su salud (e.g.: consumo de tabaco) y abandonando otras saludables (e.g.: actividad física) (Castillo, Balaguer, \& García-Merita, 2007). Estudios como los de Colley y col. (2011), Gortmaker y col. (2012) y Sanz (2017) concluyen que durante la adolescencia disminuyen los niveles de AF, aunque podría ser que este descenso comenzara desde Educación Primaria, ya que Sanz y Vinuesa (2018) obtuvieron un descenso progresivo del nivel de AF del alumnado de $3^{\circ}$ a $6^{\circ}$. Marques, González, Martins, Fernández-Villarino, y

Dirección para correspondencia [Correspondence address]: Germán Ruiz-Tendero. E-mail: german.ruiz@edu.ucm.es
Carreiro (2017) consideran que es necesario comprender qué factores influyen en los bajos niveles de práctica de AF para poder invertir dicha tendencia.

Son numerosos los determinantes que condicionan la práctica de AF. Sallis, Prochaska, y Taylor (2000), concluyeron que los más influyentes en los adolescentes (13-18 años) eran: sexo, raza, edad, orientación al logro, intención, AF previa, deportes de la comunidad, apoyo de los padres, apoyo de otras personas importantes y AF de los hermanos.

Uno de los factores más destacados es el de apoyo social, entendido como la ayuda y asistencia intercambiadas a través de las relaciones sociales y transacciones interpersonales (Heaney \& Israel, 2008). Dentro de esta red social, Heitzler y col. (2010) encontraron que, en los jóvenes, el apoyo de sus amigos era el factor más influyente en su práctica de actividad física de intensidad de moderada a vigorosa (AFMV).

El interés de los investigadores hacia el apoyo social se refleja también en las diferentes escalas que se han desarrollado a estos efectos, como por ejemplo las diseñadas por Ling, Robbins, Resnicow, y Bakhoya (2014), Prochaska, Rodgers, y Sallis (2002), Reis, Reis, y Hallal (2011), y Theodoropoulou, Karteroliotis, y Stavrou (2014). La existencia de estos instru- 
mentos permite conocer cómo influye el apoyo de familiares y amigos en los niveles de AF de los adolescentes, ya sea a través de manifestaciones de ánimo, de proporción de medio de transporte o de realización de la actividad juntos.

En base a la evidencia existente, el apoyo social que reciben los jóvenes respecto a su práctica de AF difiere en función del sexo y la edad. Lubans, Sylva, y Morgan (2007) obtuvieron que los varones recibían más apoyo de familiares y amigos que las mujeres, y que este apoyo era mayor en el grupo de menor edad (11-12 ańos) respecto del de mayor edad (1516 años). Además, hallaron correlaciones significativas entre el apoyo de familiares y amigos y, los niveles de práctica de AFMV, a excepción del apoyo de la familia a las chicas de 1112 años. Kirby, Levin, e Inchley (2011) y Cheng, Mendoça, y Farias (2014) también hallaron que los varones obtenían puntuaciones superiores a las de las mujeres.

No existe una tendencia clara respecto a si los jóvenes reciben más apoyo de familiares o de amigos para realizar AF. Norris y Ayres (2016), obtuvieron que el apoyo recibido por los jóvenes de sus familiares era superior al percibido de los amigos, en cambio, Cheng y col. (2014) obtuvieron resultados diferentes.

En base a lo expuesto y a la necesidad de conocer el estado de la cuestión en la población de estudiantes de Educación Secundaria Obligatoria de la provincia de Soria, se llevó a cabo un estudio con una triple finalidad: 1) conocer los niveles de AF diferenciando las variables de gasto energético diario (GED), gasto energético diario en actividad física de moderada a vigorosa (GED en AFMV) y, duración en AFMV en función del sexo del alumnado; 2) identificar el apoyo social percibido de familiares y amigos respecto a su práctica de AF en función del sexo del alumnado; 3) saber qué tipo de relación existe entre la práctica de AF del alumnado y el apoyo social que perciben de familiares y amigos.

\section{Método}

\section{Diseńo del estudio}

El estudio llevado a cabo es de tipo empírico ex post facto (Bisquerra \& Sabariego, 2004), epidemiológico conductual (Dishman, Heath, \& Lee, 2013) y tiene carácter descriptivo y correlacional, en base a los niveles de AF de los adolescentes de la provincia de Soria y al apoyo social que perciben de familiares y amigos respecto a dicha práctica. Además, se enmarca en el paradigma de AF y salud (Tercedor, 2001).

\section{Población y muestra}

La población objeto de estudio estuvo formada por los 3224 adolescentes que cursaban Educación Secundaria Obligatoria (ESO) en centros de la provincia de Soria. Existen dieci- nueve centros que ofertan tales estudios, nueve de los cuales se ubican en la capital, Soria. Todos los centros de la provincia excepto dos, accedieron a participar en la investigación.

El diseño muestral es de tipo no probabilístico por conveniencia, en base al criterio de captación por accesibilidad. Para ello se seleccionó un grupo de alumnos por curso y centro, favoreciendo que todos los estudiantes del mismo centro pudieran responder a los instrumentos a administrar cada uno de los días de la toma de datos.

El número de participantes encuestados fue de 1236. Una vez depurados los resultados y aplicados los criterios de exclusión que se explican a continuación, la muestra final estuvo compuesta por 694 adolescentes, cuyos descriptivos se muestran en la tabla 1.

Tabla 1. Características de la muestra.

\begin{tabular}{cccc}
\hline & & $\mathrm{n}$ & Porcentaje \\
\hline \multirow{2}{*}{ Sexo del participante } & Hombre & 364 & 52.4 \\
\cline { 2 - 4 } & Mujer & 330 & 47.6 \\
\hline \multirow{4}{*}{ Curso del participante } & 10 ESO $^{\circ}$ & 169 & 24.4 \\
& $2^{\circ}$ ESO & 179 & 25.8 \\
& $3^{\circ}$ ESO & 165 & 23.8 \\
& $4^{\circ}$ ESO & 181 & 26.1 \\
\hline \multirow{2}{*}{ Ubicación del centro } & Urbano & 321 & 46.3 \\
educativo & Rural & 373 & 53.7 \\
\hline \multirow{2}{*}{ Tipo de centro educativo } & Público & 553 & 79.7 \\
& Privado-concertado & 141 & 20.3 \\
\hline
\end{tabular}

Los criterios de exclusión de los participantes respecto de la muestra fueron: 1) no haber participado los cuatro días de administración; 2) considerar que al menos uno de los cuatro días no fue representativo respecto a lo que hace habitualmente ese día de la semana; 3) haber respondido valores atípicos.

Utilizando el software Epidat 3.1 para realizar los cálculos estadísticos de muestreo, en relación a la población y a la muestra a las que se ha hecho referencia, para un nivel de confianza de $95 \%$, y una desviación estándar de 50, se halla un error de estimación del 3.3\%.

\section{Variables}

Las variables principales de asociación son de AF y de apoyo social. La AF se descompone en: GED (METs/día), GED en AFMV (METs/día) y duración en AFMV (horas/día).

En relación al apoyo social, se diferencia el percibido de familiares y el de amigos respecto a la práctica de AF o deportiva. En relación al instrumento utilizado, las variables sobre apoyo de familiares son: 1) ánimo percibido para realizarla, 2) realización conjuntamente, 3) proporción de medio de transporte para ir a realizarla, 4) visionado durante 
la práctica, 5) comentario sobre si la estaba haciendo bien y, 6) media de los cinco ítems anteriores. Las variables sobre apoyo de amigos son: 1) ánimo emitido para que sus amigos la realicen, 2) ánimo recibido para realizarla, 3) realización conjuntamente, 4) manifestación de burlas de otros compañeros por no ser bueno realizándola; 5) comentario sobre si la estaba haciendo bien; 6) media de los cinco ítems anteriores. Todas estas variables se expresan en escala Likert de 0 a 4, a excepción de la media de los ítems que es en escala continua, tal como se explicará en el apartado posterior.

Además de presentar los resultados medios de la muestra para cada una de las variables de asociación, también se utilizará como variable independiente de agrupación el sexo del participante, diferenciando entre hombres y mujeres.

\section{Instrumentos}

Los instrumentos utilizados en la investigación han sido: Four by one-day Physical Activity Questionnaire, para medir el nivel de práctica de AF de los participantes y Parent Support Scale y Peer Support Scale, para medir el apoyo percibido de familiares y amigos respecto a su práctica de AF.

El cuestionario Four by one-day Physical Activity Questionnaire, fue diseńado y validado por Cale (1993) para participantes de más de 11 ańos. En dicho instrumento se pregunta por la AF que ha realizado cada joven el día anterior al de su administración y consta de cuatro días diferentes de respuesta. Fue adaptado y validado para población adolescente española por Cantera (1997) y Soler (2004), y validado nuevamente por Soler, Generelo, Zaragoza, y Julián (2010). El protocolo utilizado en este estudio fue el de Soler (2004). Además de en los estudios anteriores, este cuestionario ha sido utilizado en: Molinero, Martínez, Garatachea, y Márquez (2010), PeiróVelert, Valenciano, Beltrán-Carrillo, y Devís-Devís (2014) y Sanz (2018).

Las escalas de apoyo de familiares y amigos (Parent Support Scale y Peer Support Scale) fueron diseñadas por Prochaska y col. (2002). En cada escala se pregunta por cinco ítems (escala Likert de $0-4$, siendo 0 que nunca y 4 que siempre), relacionados con el apoyo percibido, los cuales corresponden a cada una de las variables citadas en el apartado anterior y la media de los ítems de esa escala.

El idioma originario de las escalas de apoyo social es el inglés, por lo que antes de utilizarse tuvieron que traducirse. Para ello se siguió el método de criterio o racional, y el modelo de traducción inversa (Cardoso, Gómez-Conesa, \& Hidalgo, 2010), basándose en la equivalencia de traducción de cada ítem realizada por dos traductoras que adaptaron el instrumento del idioma fuente al idioma objetivo, y posteriormente, otra traductora diferente volvió a traducirlo al idioma fuente, obteniendo versiones similares.

Estas escalas se administraron de forma conjunta con el cuestionario de AF, y se obtuvieron consistencias internas entre 0.7 y 0.83 , en valores de alfa de Cronbach.

\section{Procedimientos}

Se explicó el proyecto de investigación al Director y al Jefe de Programas de la Dirección Provincial de Educación de Soria, recibiendo el permiso para poder llevarlo a cabo. Posteriormente, se mantuvieron reuniones con los directores de los centros educativos que imparten cursos de ESO, y en las que se obtuvieron los permisos de acceso, se les facilitaron las copias de consentimiento informado que debían entregar al alumnado participante y que estos debían presentar antes del primer día de administración de los cuestionarios, firmado por sus padres o responsables legales.

El equipo de investigación que accedía a las aulas estaba formado por un investigador principal y de uno a cuatro investigadores ayudantes, previamente instruidos en la aplicación de los cuestionarios mediante un seminario de formación y un simulacro de encuesta, en función del número de participantes y en base al protocolo del cuestionario de AF, que establece un entrevistador por un máximo de seis entrevistados. Esta fase de administración del cuestionario y de las escalas de apoyo social se inició en enero de 2015 y finalizó en mayo del mismo año. El tiempo medio diario aproximado de administración fue de veinticinco minutos.

\section{Análisis de datos}

Para el análisis de datos se utilizó el software IBM SPSS versión 20. Primero se creó una matriz con los estadísticos obtenidos de los cuestionarios a partir de la codificación de las variables. Después, se procedió a la depuración de la matriz y, posteriormente, se aplicó el análisis estadístico definitivo.

Se hallaron los estadísticos descriptivos para las variables de estudio, mientras que la prueba $t$ de Student para muestras independientes se aplicó para la comparación de medias, observando previamente el valor de Levene $(F)$ y asumiendo varianzas no iguales $(p \leq .05)$ o iguales $(p>.05)$. En ambos casos, cuando el valor de significación de $T$ fue $p \leq .05$ se consideró que la diferencia entre las medias era significativa. De forma complementaria, se añadió el estadístico $d$ de Cohen, referido al tamaño del efecto.

Para el estudio correlacional bivariado entre las variables del nivel de AF (GED, GED en AFMV y duración en AFMV) y las puntuaciones medias de las escalas de apoyo social de familiares y amigos, incluyendo los diferentes ítems que las integran, se utilizó el coeficiente de correlación de Pearson. 


\section{Resultados}

El GED de los adolescentes de Soria es de 37.59 METs/día $(D S=3.46)$. En función del sexo, el GED de los alumnos es de $38.54 \mathrm{MET} /$ día $(D S=3.84)$ y el de las alumnas de
36.55 METs/día $(D S=2.64)$ (tabla 2). Aplicando la prueba $\mathrm{T}$ para comparar muestras independientes, $t(646,09)=7.99$ $p \leq .001$, se considera que existe diferencia estadísticamente significativa entre las medias. Además, el tamaño del efecto es moderado $(d=0.6)$.

Tabla 2. Gasto energético medio diario (METs) en función del sexo del alumnado.

\begin{tabular}{cccccc}
\hline Sexo & Media & Desviación típ. & Error típ. de la media & Mínimo & Máximo \\
\hline Masculino & 38.54 & 3.84 & .2 & 25.24 & 56.61 \\
Femenino & 36.55 & 2.64 & .15 & 27.13 & 46.67 \\
\hline
\end{tabular}

Los adolescentes de la provincia de Soria tienen un GED en AFMV de $5.93 \mathrm{METs} /$ día $(D T=4.38)$. En función del sexo, también se han obtenido valores superiores en los alumnos $(M=7.4 \mathrm{MET} /$ día; $D S=4.83)$ respecto al de las alumnas $(M=4.32 \mathrm{METs} / \mathrm{dí}$; $D S=3.12)$ (tabla 3). Aplicando las pruebas de Levene y $\mathrm{T}$ para muestras independientes, para comparar los resultados de ambas medias, se considera que existe diferencia estadísticamente significativa entre el sexo del alumnado y el GED en AFMV, pues: $t(627,91)=10.07 p$ $\leq .001$. Además, el tamaño del efecto obtenido ha sido grande $(d=0.75)$.

Tabla 3. Gasto energético medio diario (METs) en AFMV en función del sexo del alumnado.

\begin{tabular}{cccccc}
\hline Sexo & Media & Desviación típ. & Error típ. de la media & Mínimo & Máximo \\
\hline Masculino & 7.4 & 4.83 & .25 & .08 & 24.25 \\
Femenino & 4.32 & 3.12 & .17 & .17 & 18.50 \\
\hline
\end{tabular}

La duración en AFMV de los adolescentes de Soria es de 1.15 horas/día $(D S=.77)$, y en función del sexo del alumnado, se ha obtenido que los chicos la practican durante una media de $M=1.36$ horas/día $(D S=.83)$ y las chicas durante una media de $M=.93$ horas/día $(D S=.64)$ (tabla 4). Aplicando las pruebas de Levene y $\mathrm{T}$ se ha obtenido diferencia estadísticamente significativa entre las medias, pues $t(676,55)=7.66 p \leq .001$. El tamaño del efecto es $d=0.57$.

Tabla 4. Duraciones medias (horas/día) en actividades físicas moderadas-vigorosas en función del sexo del alumnado.

\begin{tabular}{cccccc}
\hline Sexo & Media & Desv.típ. & Error típ. media & Mínimo & Máximo \\
\hline Masculino & 1.36 & .83 & .04 & .02 & 4.93 \\
Femenino & .93 & .64 & .04 & .02 & 3.54 \\
\hline
\end{tabular}

La valoración media obtenida por los alumnos en la escala de apoyo percibido de los miembros de su casa es de $M=2.15$ $(D S=.82)$, superior a la de las alumnas que es de $M=2.03$ $(D S=.8)$. En ambos casos, se obtuvieron similares valores tanto mínimos (0) como máximos (4) (tabla 5).

En dicha escala, el ítem con peor puntación media, independientemente del sexo del alumno, ha sido el referido a recibir ánimos durante la práctica de AF. Se han obtenido diferencias estadísticamente significativas entre las medias de las siguientes variables: miembros de su familia le han proporcionado medios de transporte para realizar AF $(t(692)=$ $3.85 p \leq .001)$, miembros de su familia le han visto realizar AF $(t(692)=2.17 p \leq .05)$ y, puntuación media total de la escala de apoyo de familiares $(t(692)=2.01 p \leq .05)$. Además, los tamaños del efecto han sido: $d=0.3 ; d=0.17$; $d=0.15$, respectivamente. 
Tabla 5. Valoraciones medias del alumnado sobre el apoyo percibido de los miembros de su casa en función de su sexo.

\begin{tabular}{cccccccc}
\hline \multirow{2}{*}{ Sexo } & & $\begin{array}{c}\text { Te han } \\
\text { animado }\end{array}$ & $\begin{array}{c}\text { Habéis hecho } \\
\text { AF juntos }\end{array}$ & $\begin{array}{c}\text { Proporcionan trans- } \\
\text { porte }\end{array}$ & $\begin{array}{c}\text { Te vieron } \\
\text { hacer AF }\end{array}$ & $\begin{array}{c}\text { Te dijeron que } \\
\text { lo hacías bien }\end{array}$ & $\begin{array}{c}\text { Media de los } \\
\text { ítems }\end{array}$ \\
\hline \multirow{4}{*}{ Alumnos } & Media & 2.19 & 1.61 & 2.36 & 2.26 & 2.34 & 2.15 \\
& Desv. típ. & .89 & .9 & 1.17 & 1.03 & 1.06 & .82 \\
& E. típ. med. & .05 & .05 & .06 & .05 & .06 & .04 \\
\multirow{4}{*}{ Alumnas } & Media & 2.17 & 1.65 & 2.01 & 2.09 & 2.2 & 2.03 \\
& Desv. típ. & .87 & .88 & 1.18 & .94 & 1.04 & .06 \\
\multirow{4}{*}{ Total } & E. típ. med. & .05 & .05 & .06 & .05 & .06 & .04 \\
& Media & 2.18 & 1.63 & 2.19 & 2.18 & 2.27 & 2.09 \\
& Desv. típ. & .88 & .89 & 1.18 & .99 & 1.05 & .81 \\
\hline
\end{tabular}

La valoración media de los alumnos en la escala sobre apoyo de los amigos ha sido de $M=2.15(D S=.72)$ y la de las alumnas ha sido de $M=1.55(D S=.59)$ (tabla 6$)$.

Los ítems que han obtenido peores y mejores puntuaciones medias han sido los mismos en ambos sexos, concretamente los referidos a si los amigos se burlan de ellos y a si realizan $\mathrm{AF}$ juntos, respectivamente.

Se han obtenido diferencias estadísticamente significati- vas entre las medias de las siguientes variables en función del sexo: otros nińos les han animado a realizar AF $(t(692)=4.89$ $p \leq .001)$, otros niños les han dicho que estaban haciendo bien la AF $(t(692)=2.37 p \leq .01)$, y puntuación media total de la escala de apoyo de amigos $(t(692)=2.73 p \leq .01)$. Los tamaños del efecto han sido: $d=0.08, d=0.18 \mathrm{y}, d=0.21$, respectivamente.

Tabla 6. Valoraciones medias del alumnado sobre el apoyo percibido de sus amigos en función de su sexo

\begin{tabular}{|c|c|c|c|c|c|c|c|}
\hline \multicolumn{2}{|c|}{ Sexo } & $\begin{array}{l}\text { Les animas a } \\
\text { realizar AF }\end{array}$ & $\begin{array}{l}\text { Te animan a } \\
\text { realizar AF }\end{array}$ & $\begin{array}{l}\text { Realizáis AF } \\
\text { juntos }\end{array}$ & $\begin{array}{c}\text { Otros niños se } \\
\text { burlan de ti }\end{array}$ & $\begin{array}{c}\text { Te dijeron que lo } \\
\text { hacías bien }\end{array}$ & $\begin{array}{l}\text { Media de } \\
\text { los ítems }\end{array}$ \\
\hline \multirow{3}{*}{ Alumnos } & Media & 2.05 & 1.89 & 2.65 & .29 & 2.02 & 1.67 \\
\hline & Desv. típ. & .9 & .88 & .86 & .57 & .89 & .57 \\
\hline & E. típ. med. & .05 & .05 & .05 & .03 & .05 & .03 \\
\hline \multirow{3}{*}{ Alumnas } & Media & 1.99 & 1.82 & 2.33 & .27 & 1.86 & 1.55 \\
\hline & Desv. típ. & .79 & .85 & .88 & .6 & .92 & .59 \\
\hline & E. típ. med. & .04 & .05 & .05 & .03 & .05 & .03 \\
\hline \multirow{2}{*}{ Total } & Media & 2.02 & 1.86 & 2.5 & .28 & 1.95 & 1.61 \\
\hline & Desv. típ. & .85 & .87 & .88 & .58 & .91 & .58 \\
\hline
\end{tabular}

En relación a las correlaciones entre las variables de AF (GED, GED en AFMV y duraciones de AFMV), con las variables de las escalas de apoyo social de familiares y amigos (tablas 7 y 8), los resultados obtenidos son de forma predominante estadísticamente significativos, siéndolo a nivel $p \leq .001$ (bilateral), teniendo relaciones positivas y con intensidades de asociación débiles $(r \leq .5)$. La única variable de apoyo social en la que no se han obtenido correlaciones estadísticamente significativas ha sido la relativa a si habían percibido burlas de otros iguales respecto a su práctica de AF, además, la correlación obtenida es negativa.

Tabla 7. Relaciones entre la práctica de actividad física y el apoyo social de los familiares (prueba r de Pearson).

\begin{tabular}{|c|c|c|c|c|c|c|}
\hline & $\begin{array}{l}\text { Le animan a hacer } \\
\text { AF }\end{array}$ & $\begin{array}{l}\text { Hacen AF } \\
\text { juntos }\end{array}$ & $\begin{array}{l}\text { Le propor. trans- } \\
\text { porte }\end{array}$ & Ver hacer AF & $\begin{array}{c}\text { Decir AF bien } \\
\text { hecha }\end{array}$ & $\begin{array}{l}\text { Media ítems } \\
\text { escala }\end{array}$ \\
\hline GED & $.15^{* *}$ & $.11^{*}$ & $.29^{* *}$ & $.29^{* *}$ & $.21^{* *}$ & $.27^{* *}$ \\
\hline GED AFMV & $.14^{* *}$ & $.14^{* *}$ & $.32^{* *}$ & $.34^{* *}$ & $.26^{*}$ & $.3^{* *}$ \\
\hline Duración AFMV & $.16^{* *}$ & $.14^{* *}$ & $.28^{* *}$ & $.29^{* *}$ & $.24^{* *}$ & $.28^{* *}$ \\
\hline
\end{tabular}

Nota. *. La correlación es significativa al nivel .01 (bilateral). ${ }^{* *}$. La correlación es significativa al nivel .001 (bilateral). 
Tabla 8. Relaciones entre la práctica de actividad física y el apoyo social de los amigos (prueba $\mathrm{r}$ de Pearson).

\begin{tabular}{|c|c|c|c|c|c|c|}
\hline & $\begin{array}{c}\text { Animan a } \\
\text { hacer AF }\end{array}$ & $\begin{array}{c}\text { Son animados } \\
\text { a } \mathrm{AF}\end{array}$ & $\begin{array}{l}\text { Realizar AF } \\
\text { juntos }\end{array}$ & $\begin{array}{c}\text { Se burlan de ellos } \\
\text { por AF }\end{array}$ & $\begin{array}{c}\text { Les dijeron AF } \\
\text { bien }\end{array}$ & $\begin{array}{l}\text { Media ítems } \\
\text { escala }\end{array}$ \\
\hline GED & $.27^{* *}$ & $.18^{* *}$ & $.42^{* *}$ & -.02 & $.27^{* *}$ & $.35^{* *}$ \\
\hline GED AFMV & $.25^{* *}$ & $.12^{* *}$ & $.44^{* *}$ & -.05 & $.29^{* *}$ & $.34^{* *}$ \\
\hline Duración AFMV & $.24^{* *}$ & $.1^{*}$ & $.4^{* *}$ & -.06 & $.29^{* *}$ & $.33^{* *}$ \\
\hline
\end{tabular}

Nota. *. La correlación es significante al nivel .01 (bilateral). **. La correlación es significativa al nivel .001 (bilateral).

\section{Discusión}

En relación a la $\mathrm{AF}$, los resultados hallados en función del sexo del alumnado están en consonancia con los de otros estudios, siendo los niveles de GED, GED en AFMV y duración en AFMV de los varones significativamente superiores a los de las mujeres. Resultados similares obtuvieron Cale (1993), Cantera (1997), y Peiró-Velert y col. (2014), utilizando el mismo instrumento de medida de la actividad física $y$, Colley y col. (2011), Gortmaker y col. (2012), Sanz (2017) y Torres-Luque, Calahorro, López,Fernández y Carnero (2014), administrando otro distinto.

En este estudio se obtuvieron diferencias estadísticamente significativas entre las medias de las escalas de apoyo social de los adolescentes, siendo superior en los varones de Soria, resultados similares a los de Cheng, Mendoça, y Farias Júnior (2014), Gontarev, Kalac, Ameti, y Redjepi (2016) y Norris y Ayres (2016). Además, la superioridad obtenida en este estudio se mantiene en todos los ítems de las escalas excepto en el referido a realizar AF con familiares, que es superior en las alumnas.

Las correlaciones halladas entre los niveles de AF y el apoyo percibido de familiares y amigos, consolidan este como uno de los determinantes que tiene más relación con la AF de los adolescentes. Esto, no solo está en consonancia con lo aportado por Sallis y col. (2000), sino que, además, se han obtenido relaciones estadísticamente significativas con la mayoría de los ítems en que se han diferenciado las percepciones del apoyo.

En base a la evidencia existente y a la hallada en el presente estudio, sería conveniente que las chicas de Educación Secundaria Obligatoria de la provincia de Soria gozaran de mayor apoyo de familiares y amigos respecto a su práctica de AF, ya que es un grupo tendente a alcanzar bajos niveles de práctica y a convertirse en inactivo. Además, se aconseja realizar propuestas de intervención para aumentar los niveles de AF del alumnado en general y, de las mujeres en particular.

El apoyo que perciban los jóvenes de familiares estará relacionado positivamente con sus niveles de AF presentes $y$, estos a su vez pueden hacerlo en los de etapas evolutivas posteriores, como en la adultez (Bélanger, Sabiston, Barnett, O'Loughlin, Ward, Contreras, \& O'Loughlin, 2015). Lo mismo ocurrirá con el apoyo de amigos, ya que Heitzler y col. (2010) concluyeron que era el factor con mayor relación respecto a la práctica de AFMV de los jóvenes.

En el estudio se asumen las limitaciones derivadas del componente de respuesta subjetiva y pérdida de muestra por defectos en su compleción, propias del instrumento de medida de la AF utilizado y, del sesgo de selección, debido a la realización de un diseño no probabilístico por conveniencia.

Algunas de las futuras líneas de investigación a tener en cuenta serían realizar un estudio epidemiológico conductual de tipo longitudinal, para conocer cómo evolucionan los niveles de AF y de apoyo social con el paso del tiempo y, llevar a cabo investigaciones para conocer los niveles de AF de la población soriana utilizando instrumentos de mayor objetividad.

Los resultados hallados en esta investigación nos permiten concluir que: 1) los niveles de AFMV del alumnado adolescente de la provincia de Soria son bajos, especialmente los de las chicas; 2) el apoyo que los adolescentes perciben de su familia es superior al percibido de sus amigos; 3) existen diferencias en los niveles de AF en función del sexo del alumnado, siendo superiores en los varones; 4) existe una relación estadísticamente significativa entre el apoyo social percibido por los alumnos y su nivel de AF.

\section{Referencias}

1. Bélanger, M., Sabiston, C. M., Barnett, T. A., O’Loughlin, E., Ward, S., Contreras, G., \& O'Loughlin, J. (2015). Number of years of participation in some, but not all, types of physical activity during adolescence predicts level of physical activity in adulthood: Results from a 13-year study. International Journal of Behavioral Nutrition and Physical Activity, 12(76). doi: 10.1186/s12966-015-0237-x

2. Bisquerra, R., \& Sabariego, M. (2004). Fundamentos metodológicos de la investigación educativa. En R. Bisquerra (Coord.) (2004). Metodologia de la investigación educativa (pp. 19-49). Madrid: La Muralla.
3. Cale, L. (1993). Monitoring Physical Activity in children (Tesis doctoral). Recuperado de Loughborough University Institutional Repository.

4. Cantera, M. A. (1997). Niveles de actividad física en la adolescencia. Estudio realizado en la población escolar de la provincia de Teruel (Tesis doctoral). Universidad de Zaragoza, Huesca.

5. Cardoso, C., Gómez-Conesa, A., \& Hidalgo, M. D. (2010). Metodología para la adaptación de instrumentos de evaluación. Fisioterapia, 32(6), 264-270. doi: 10.1016/j.ft.2010.05.001 
6. Castillo, I., Balaguer, I., \& García-Merita, M. (2007). Efecto de la práctica de actividad física y de la participación deportiva sobre el estilo de vida saludable en la adolescencia en función del género. Revista de Psicología del Deporte, 16(2), 201-210.

7. Cheng, L. A., Mendoça, G., \& Farias Júnior, J. C. (2014). Physical activity in adolescents: Analysis of the social influence of parents and friends. Jornal de Pediatria (Rio de Janeiro), 90(1), 35-41. doi: 10.1016/j. jped.2013.05.006

8. Colley, R. C., Garriguet, D., Janssen, I., Craig, C. L., Clarke, J., \& Tremblay, M. S. (2011). Physical activity of Canadian children and youth: Accelerometer results from the 2007 to 2009 Canadian Health Measures Survey. Health Reports, 22(1), 15-23.

9. Dishman, R. K., Heath, G. W., \& Lee, I. (2013). Physical Activity Epidemiology. Champaign: Human Kinetics.

10. Gontarev, S., Kalac, R., Ameti, V., \& Redjepi, A. (2016). Factors Associated with Physical Activity among Macedonian Adolescents in Albanian Ethnic Community. Iranian Journal of Public Health, 45(4), 474-484.

11. Gortmaker, S. L., Lee, R., Cradock, A. L., Sobol, A. M., Duncan, D. T., \& Wang, Y. C. (2012). Disparities in Youth Physical Activity in the United States: 2003-2006. Medicine \& Science in Sports \& Exercise, 44(5), 888-893. doi: 10.1249/MSS.0b013e31823fb254.

12. Heaney, C. A., \& Israel, B. A. (2008). Social Networks and Social Support. En K. Glanz, B. K. Rimer y K. Viswanath (Eds.) Health Behavior and Health Education: Theory, Research and Practice (pp.189-210). San Francisco: Jossey-Bass.

13. Heitzler, C. D., Lytle, L. A., Erickson, D. J., Barr-Anderson, D., Sirard, J. R., \& Story, M. (2010). Evaluating a Model of Youth Physical Activity. American Journal of Health Behavior, 34(5), 593-606.

14. Kirby, J., Levin, K. A., \& Inchley, J. (2011). Parental and Peer Influences on Physical Activity Among Scottish Adolescents: A Longitudinal Study. Journal of Physical Activity and Health, 8(6), 785 -793. doi: 10.1123/jpah.8.6.785

15. Ling, J., Robbins, L. B., Resnicow, K., \& Bakhoya, M. (2014). Social Support and Peer Norms Scales for Physical Activity in Adolescents. American Journal of Health Behavior, 38(6), 881-889. doi:10.5993/ AJHB.38.6.10.

16. Lubans, D. R., Sylva, K., \& Morgan, P. J. (2007). Factors associated with physical activity in a sample of British secondary school students. Australian Journal of Educational \& Developmental Psychology, 7, 22-30.

17. Marques, A., González, M., Martins, J., Fernández-Villarino, M. A., \& Carreiro, F. (2017). Relación entre la actividad física de los adolescentes y la de madres/ padres. Revista de Psicología del Deporte 26(1), 145-156.

18. Molinero, O., Martínez, R., Garatachea, N., \& Márquez, S. (2010). Pautas de actividad física de adolescentes españolas: Diferencias mediadas por la participación deportiva y el día de la semana. Revista de Psicología del Deporte, 19(1), 103-116.

19. Norris, S. M., \& Ayres, C. G. (2016). Factors influencing the health promoting physical activity behaviors of diverse urban adolescents.
Journal of Nursing Practice Applications \& Reviews of Research, 6(1), 1623. doi: 10.13178/jnparr.2016.0601.0316

20. Organización Mundial de la Salud (OMS). (2009). Global health risks: mortality and burden of disease attributable to selected major risks. Ginebra: OMS.

21. Organización Mundial de la Salud (OMS). (2010). Global recommendations on physical activity for health. Ginebra: OMS.

22. Peiró-Velert, C., Valenciano, J., Beltrán-Carrillo, V., \& Devís-Devís, J. (2014). Variabilidad de la actividad física en adolescentes españoles de 17-18 años en función del tipo de jornada y época del año. Revista de Psicología del Deporte, 23(2), 347-354.

23. Prochaska, J. J., Rodgers, M. W., \& Sallis, J. F. (2002). Association of parent and peer support with adolescent physical activity. Research Quarterly for Exercise and Sport, 73(2), 206-210.

24. Reis, M. S., Reis, R. S., \& Hallal, P. C. (2011). Validity and reliability of a physical activity social support assessment scale. Revista de Saúde Pública, 45(2), 294-301.

25. Sallis, J. F., Prochaska, J. J., \& Taylor, W. C. (2000). A review of correlates of physical activity of children and adolescents. Medicine \& Science in Sports \& Exercise, 32(5), 963-975.

26. Sanz, D. (2017). Niveles de actividad física moderada-vigorosa de adolescentes del municipio de Soria. Sportis Scientific Journal, 3(1), 100 122. doi: 10.17979/sportis.2017.3.1.1738

27. Sanz, D. (2018). Niveles de actividad física y apoyo social percibido del alumnado adolescente de la provincia de Soria (Tesis doctoral). Universidad Complutense de Madrid, Madrid.

28. Sanz, D., \& Vinuesa, R. (2018). Relación entre los niveles de actividad física y los rasgos definitorios de roles de situaciones de agresiónvictimización en escolares. Sportis Scientific Journal, 4(1), 59-76-. doi: 10.17979/sportis.2018.4.1.2065

29. Soler, J. J. (2004). Análisis de la frecuencia cardiaca registrada en las clases de Educación Física y su relación con los niveles de actividad física diaria de un grupo de alumnos de la educación secundaria obligatoria. Implicaciones para la salud y la labor docente (Tesis Doctoral). Universidad de Zaragoza, Zaragoza.

30. Soler, J. J., Generelo, E., Zaragoza, J., \& Julián, J. A. (2010). Validez de criterio y confiabilidad del "Four by One Day Physical Activity Questionnaire" en población adolescente española. Apunts. Educación Física y Deportes, 101(3), 19-24.

31. Tercedor, P. (2001). Actividad física, condición física y salud. Sevilla: Wanceulen.

32. Theodoropoulou, E., Karteroliotis, K., \& Stavrou, N. (2014). Validity and Reliability of Greek Versions of Two Scales Assessing Family and Friend Support for Exercise Behaviour. Perceptual and Motor Skills, 118(1), 26-40. doi:10.2466/06.08.PMS.118k13w7

33. Torres-Luque, G., Calahorro, F., López,Fernández, I., \& Carnero, E. A. (2014). Impact of gender on levels of physical activity in physical education. Sport TK: Revista Euroamericana de Ciencias del Deporte, 3(1-2), 21-26. doi: 10.6018/221971 